\title{
Sleep quality and anxiety among nurses in tertiary hospitals in China and the influencing factors: $A$ cross-sectional study
}

Dongbo Wang

Beijing Tongren Hospital

Jing-Lei Lian

Capital Medical University Affiliated Anzhen Hospital

Ming-Rui Hua

Beijing Tongren Hospital

Yu-Da Pan

Capital Medical University

De-Min Han ( $\nabla$ enthandm@126.com )

Research article

Keywords: Sleep disorder, Anxiety, Quality of life, Nurses, Influencing factor

Posted Date: April 8th, 2020

DOI: https://doi.org/10.21203/rs.3.rs-17774/v1

License: (1) (i) This work is licensed under a Creative Commons Attribution 4.0 International License.

Read Full License 


\section{Abstract}

\section{Background}

The purpose of the study was to investigate to understand the situation of sleep quality and anxiety among nurses in tertiary hospitals in China, and analyze their influencing factors.

\section{Methods}

A cross-sectional survey was performed for nurses in Beijing tertiary hospitals including Beijing Tongren hospital, Beijing Anzhen Hospital and Beijing Children Hospital. The Pittsburgh Sleep Quality Indes (PSQI) and Zung Self-Rating Anxiety Scale (SAS) were used to measured the sleep quality and anxiety symptoms, respectively. Survey of quality of life in nurses was measured by 36-item Short Form Health Survey (SF-36). Spearman's correlations analysis and logistic regression analysis was used to understand the influencing factors with sleep disorder, anxiety symptoms and quality of sleep. Quality of life (both PCS and MCS) were affected by influencing factors including marital status, working years, education background, income monthly, working hours, regular diet, physical exercise, sleep disorder, anxiety symptoms, felling of stress, and stress from economic, social, occupational.

Results

643 registered nurses in three tertiary hospitals were surveyed, $517(80.4 \%)$ returned questionnaires were valid for analysis. The average PSQI score was $7.71 \pm 3.62$, including 372 participants that scores were above 5 (72.0\%). The average SAS score was $45.18 \pm 9.90$,including 157 participants with a SAS score > $50(30.4 \%)$ that had different anxiety symptoms. The nurses' sleep quality were affected by some factors including income monthly, working hours, regular diet, physical exercise, stress from economic, social, occupational. Education background, income monthly, working hours, regular diet and economic stress, social stress, occupational stress have significantly related to anxiety symptoms. Correlation between sleep disorder and anxiety severity was positive for nurses.

\section{Conclusions}

The situation of sleep disorder and anxiety symptoms among nurses in tertiary hospitals in China were very serious. The problem has negative effect on physical and mental health in nurses which remind hospital administrators should take preventive actions. In addition, the results of the study hinted that promotion of health lifestyle, effort-reward balance and providing social support may be significant to decrease the poor sleep quality and anxiety symptoms and increase quality of life in nurses.

\section{Background}

The status of physical and mental health have important effect for quality of life and work for medical staffs[1]. Clinical nurses, as an important medical group, maintain faster work pace and heavier workload for a long time that increase the risk of physical and mental health problem, including feeling stress, 
fatigue, burnout, anxiety, and decreasing the quality of life. However, as a negative result, poor sleep quality has becoming an obvious and complaint problem for nursing staff all over the world[2-5]. Shift work disorder, stressful workload and complex work environment had been studied that may be the main reasons of sleep problems for nurses[2, 3, 4, 6].Poor sleep quality has negative effects for physical health such as metabolic disturbance, cardiometabolic disorders and chronic diseases[6-10].

Moreover, sleep problems for a long time also could result in serious effects for psychology and mental health including thought retardation, low spirit, irritability, depression and so on[11, 12]. In fact, apart from impacting health of nurses, the previous studies have been improved that poor sleep quality could decrease the work performance and increase the risk of medical errors, impaired psychomotor performance over the past 40 years[13-18].

As we know, anxiety is an unpleasant emotion characterized by fear, worry, rumination[19].However, anxiety disorder is medical disease if it was persistent and intense[19]. Anxiety disorder has become a worldwide problem that we need focus on. Previous researches reported that one-eighth population of world may suffer from inappropriate anxiety[20].Some influencing factors were supposed like women,older age, urban,obese,and so on[21-22]. Previous studies also have indicated that nurses was a higher risk professional group that with anxiety disorder that others, $21 \%$ of Singapore general hospital nurses, $20 \%$ of ICU and general care nurses in the US, $43.2 \%$ of shift-work nurses in Iranian have suffered from anxiety disorder, respectively[23-25].

Chinese hospitals are divided into three degrees(tertiary hospital;sencod-class hospital; class-I hospital) according to hospital scale, clinical staffs, work quality, technical facilities, etc. As of 2016, there were 2232 tertiary hospital(7.6\% all kinds of hospitals in China). In 2016, there were 2213718 hospital beds(the proportion of all is $43.97 \%$, this number was $40.71 \%$ in 2012 ) in Chinese tertiary hospitals and there were 1246308 registered nurses; and have provided high-quality medical care for 1627848 thousands of patients (the proportion of all is $49.78 \%$, this number was $42.75 \%$ in 2012)[26]. As we know, tertiary hospital represents the highest medical care level in China, Chinese patients would like obtaining their clinical care no matter haw far and how long time spent in waiting. Therefore, nurses in Chinese tertiary hospitals were faced with heavy workload, stressful work environment and complex interpersonal relationship. Chinese studies indicated that the quality of life of nurses in Chinese tertiary hospital was facing great challenge[6, 27-28].

Previous researches have shown that we should pay more attention to physical and mental health of clinical nurses.Nurses are encountering more stressful job and workload in tertiary hospital than that in secondary and first-level hospitals. Not only that, they were more possibility and risk for physical and mental health and medical errors. However, we do not know that clearly. Then, the purpose of our study was understanding the status and influencing factors of sleep quality, source of stress, mental health, quality of life and their correlation among nurses in tertiary hospitals in China. We also hope to offer some suggestion for improving their quality of life and work. 


\section{Methods \\ Participants}

We selected three famous tertiary hospitals in China locating in Beijing into our cross-sectional survey that a lot of patients all over China were coming because of their high quality of medical care, including Beijing Children hospital,Beijing Tongren Hospital, and Anzhen hospital. We have surveyed 643 nurses by self-administered questionnaire from April to June in 2018, and 517 questionnaires were returned valid(80.4\%). These 517 nurses were becoming our participants in our study.

\section{Survey Questionnaire}

Our self-administered questionnaire consisted of five parts, including survey of basic information, source of stress, quality of sleep, anxiety and quality of life.

\section{Basic Information And Source Of Stress}

The basic information of study contained sex; age; marital status; seniority; education level (junior college degree or below, bachelor degree or above); professional status ( primary, intermediate or above); monthly income; working hours per week, smoking, drinking, regular diet and physical exercise. We understood the subjective feeling of source of stress by the question "what do you think the main source of your stress? Economic stress, occupational stress, social stress, or other reason".

\section{Quality Of Sleep}

The Pittsburgh Sleep Quality Index(PSQI) is a valid and widely instrument to measure and assess the quality of sleep for the general and clinical populations over a 1-month period[29, 30]. The Chinese version of Pittsburgh Sleep Quality Index(C-PSQI) had an good reliability and sensitivity using cutoff value of 5 that discriminate poor from good quality of sleep[30]. C-PSQI contain 19 items that are combined into 7 component scores including subjective sleep quality, sleep latency, sleep duration, habitual sleep efficiency, sleep disturbance, used sleep medication, daytime dysfunction[30]. The range of global score is from 0 to 21 summing 7 component scores, and higher scores represent the subjective quality of sleep is poorer[6, 29, 30].

\section{Anxiety}

We measured the anxiety symptoms of our participants by the Zung Self-Rating Anxiety Scale(SAS) that has an good internal consistency and test-retest reliability[31-33]. There are 20 items gathered the scale, and every item has 4 options that scored different scores. The range of total raw score is from 20 to 80 ,or 
index score is from 25 to 100.The upper limit for the normal was a raw score 40 or index score 50 , respectively. A higher score indicate higher level of anxiety symptoms. The study used the index score to measure the anxiety symptom. When the total index score $\geq 50$ was defined as "mild anxiety symptom", $\geq 60$ that was moderate anxiety, $\geq 70$ was severe anxiety according to Chinese version[34].

\section{Quality Of Life}

In the study, we selected the 36-item Short Form Health Survey (SF-36) to evaluate the quality of life (QOL) for our participants. The SF-36 is also a reliable and effective measuring tool to assess QOL of patients and occupational populations [35-36]. The measurement contents of 8 dimensions constitute the scale, including physical function (PF), role physical (RP), bodily pain (BP), global health (GH), vitality $(\mathrm{VT})$, social function(SF), role emotional (RE), mental health(MH). The range of scores of every dimension is from 0 to 100. The higher score represents the better possible health status. The scores of eight dimensions are summarized in physical component summary (PCS) and mental component summary (MCS) scores.

\section{Statistical Analyses}

Firstly, we understood the status of quality of sleep, source of stress, anxiety and quality of life among our participants by descriptive statistical analysis. Secondly, Spearman's correlations analysis was used to compare the scores of PSQI and SAS and the t-test and the analysis of variance (ANOVA) were used to compare the scores of SF-36 (the scores of PCS and MCS) among the factors about demographic characteristics, lifestyle, and felling of stress. The t-test was used to compare the scores of SF-36(PCS and MCS) between whether there is sleep disorder, and the analysis of variance (ANOVA) was used to compare that among different anxiety severity groups. Thirdly, Spearman's correlations analysis was used to understand the relationship between sleep disorder and anxiety severity. Finally, logistic regression analysis was using to explore factors associated with sleep disorder and anxiety symptoms. SPSS version 20.0 was used to analysis the date in the study. All tests accepted the $5 \%$ significance level.

\section{Results}

\section{Characteristics of participants}

All the participants of our study were female, and their average age was $30.49 \pm 7.53$ years. The average PSQI score was $7.71 \pm 3.62$, including 372 participants that scores were above 5 (72.0\%). The average SAS score was $45.18 \pm 9.90$,including 157 participants with a SAS score $\otimes 50(30.4 \%)$ that had different anxiety symptoms. According our results, there were 334 nurses (64.6\%) felling heavy or very heavy stress, and 355 nurses(68.7\%) felling economic stress, 407 nurses $(78.7 \%)$ felling occupational stress and 249 nurses $(48.2 \%)$ felling social stress. 


\section{Influencing Factors Analysis Related To Quality Of Sleep}

Table 1 showed the results that different factors affected the quality of sleep. The nurses' sleep quality were affected by some factors including income monthly, working hours, regular diet, physical exercise, stress from economic, social, occupational. However, we did not find the marital status, working years, education background, professional title, leader, smoking and alcohol consumption were significantly related to quality of sleep.

\section{Influencing Factors Analysis Related To Anxiety Symptoms}

Results of influencing factors analysis showed education background, income monthly, working hours, regular diet and economic stress, social stress, occupational stress have significantly related to anxiety symptoms. Whereas marital status, working years, professional title, leader, smoking, alcohol consumption and physical exercise were not. The results were showed in Table 2.

\section{Influencing Factors Analysis Related To Quality Of Life}

Table 3 indicated quality of life (both PCS and MCS) were affected by influencing factors including marital status, working years, education background, income monthly, working hours, regular diet, physical exercise, sleep disorder, anxiety symptoms, felling of stress, and stress from economic, social, occupational. However, smoking, alcohol consumption and leader were not. Professional title was significant related to MCS, but not to PSC.

\section{The Relationship Between Sleep Disorder And Anxiety Severity}

Correlation between sleep disorder and anxiety severity was positive for nurses. The result was showed in Table 4.

\section{Logistic Regression Analysis Of Multiple Factors Influencing Sleep Disorder}

Table 5 showed that physical exercise, regular diet and income monthly have independent influence with sleep disorder for nurses. In other words, physical exercise frequency, keeping regular diet and salary suitably were positive factors for preventing sleep disorder for nurses. 


\section{Logistic Regression Analysis Of Multiple Factors Influencing Anxiety Symptoms}

Table 6 showed that anxiety symptoms revealed an independent relevance with education background, working hours, regular diet, economic stress. In other words, the risk factors for anxiety in nurses were higher education background, longer working hours, irregular diet and economic stress. 
Table 1

Influencing factors analysis related to quality of sleep ( $N=517)$

\begin{tabular}{|c|c|c|c|c|}
\hline \multicolumn{2}{|c|}{ Demographic characteristics } & \multirow{2}{*}{$\begin{array}{l}\text { Number of participants } \\
\text { without Sleep disorder } \\
75\end{array}$} & \multirow{2}{*}{$\begin{array}{l}\text { Number of } \\
\text { participants with } \\
\text { sleep disorder } \\
223\end{array}$} & \multirow{2}{*}{$\begin{array}{l}P \\
\text { value } \\
0.089\end{array}$} \\
\hline Marital status & Married/cohabitation & & & \\
\hline & $\begin{array}{l}\text { Unmarried/divorced } \\
\text { /widowed }\end{array}$ & 70 & 149 & \\
\hline \multirow[t]{5}{*}{ Working years } & $\leq 5$ years & 64 & 130 & \multirow[t]{5}{*}{0.288} \\
\hline & $6-10$ years & 20 & 75 & \\
\hline & $11-15$ years & 24 & 70 & \\
\hline & $15-20$ years & 14 & 35 & \\
\hline & $\nabla 20$ years & 23 & 62 & \\
\hline \multirow[t]{2}{*}{$\begin{array}{l}\text { Education } \\
\text { background }\end{array}$} & $\begin{array}{l}\text { Junior college } \\
\text { or below }\end{array}$ & 64 & 145 & \multirow[t]{2}{*}{0.283} \\
\hline & $\begin{array}{l}\text { Bachelor degree or } \\
\text { above }\end{array}$ & 81 & 227 & \\
\hline \multirow{2}{*}{$\begin{array}{l}\text { Professional } \\
\text { title }\end{array}$} & Junior & 105 & 244 & \multirow[t]{2}{*}{0.137} \\
\hline & $\begin{array}{l}\text { Medium-grade or } \\
\text { above }\end{array}$ & 40 & 128 & \\
\hline \multirow[t]{2}{*}{ leader } & Yes & 9 & 14 & \multirow[t]{2}{*}{0.226} \\
\hline & No & 136 & 358 & \\
\hline \multirow{3}{*}{$\begin{array}{l}\text { Income } \\
\text { monthly(RMB) }\end{array}$} & $<5000$ & 37 & 31 & \multirow[t]{3}{*}{0.000} \\
\hline & $5000-10000$ & 99 & 325 & \\
\hline & 10000 & 9 & 16 & \\
\hline \multirow{3}{*}{$\begin{array}{l}\text { Working hours } \\
\text { a week }\end{array}$} & $<40 \mathrm{H}$ & 88 & 172 & \multirow[t]{3}{*}{0.000} \\
\hline & $41-56 \mathrm{H}$ & 55 & 190 & \\
\hline & $\geq 56 \mathrm{H}$ & 2 & 9 & \\
\hline \multirow[t]{2}{*}{ Smoking } & Yes & 2 & 12 & \multirow[t]{2}{*}{0.244} \\
\hline & No & 143 & 359 & \\
\hline
\end{tabular}




\begin{tabular}{|c|c|c|c|c|}
\hline \multicolumn{2}{|c|}{ Demographic characteristics } & \multirow{2}{*}{$\begin{array}{l}\text { Number of participants } \\
\text { without Sleep disorder } \\
8\end{array}$} & \multirow{2}{*}{$\begin{array}{l}\text { Number of } \\
\text { participants with } \\
\text { sleep disorder } \\
24\end{array}$} & \multirow{2}{*}{$\begin{array}{l}P \\
\text { value } \\
0.701\end{array}$} \\
\hline $\begin{array}{l}\text { Alcohol } \\
\text { consumption }\end{array}$ & Yes & & & \\
\hline & No & 134 & 342 & \\
\hline \multirow[t]{3}{*}{ Regular diet } & Yes & 85 & 142 & \multirow[t]{3}{*}{0.000} \\
\hline & General & 52 & 168 & \\
\hline & no & 8 & 62 & \\
\hline \multirow[t]{3}{*}{$\begin{array}{l}\text { Physical } \\
\text { exercise }\end{array}$} & $\begin{array}{l}3 \text { or more times a } \\
\text { week }\end{array}$ & 21 & 14 & \multirow[t]{3}{*}{0.000} \\
\hline & 1-2 times a week & 41 & 98 & \\
\hline & No & 83 & 260 & \\
\hline \multirow{2}{*}{$\begin{array}{l}\text { Economic } \\
\text { stress }\end{array}$} & No & 55 & 107 & \multirow[t]{2}{*}{0.044} \\
\hline & Yes & 90 & 265 & \\
\hline \multirow{2}{*}{$\begin{array}{l}\text { Occupational } \\
\text { stress }\end{array}$} & No & 41 & 69 & \multirow[t]{2}{*}{0.000} \\
\hline & Yes & 104 & 303 & \\
\hline \multirow[t]{2}{*}{ Social stress } & No & 86 & 182 & 0.034 \\
\hline & Yes & 59 & 190 & \\
\hline
\end{tabular}


Table 2

Influencing factors analysis related to anxiety symptoms $(\mathrm{N}=517)$

\begin{tabular}{|c|c|c|c|c|}
\hline \multicolumn{2}{|c|}{ Demographic characteristics } & \multirow{2}{*}{$\begin{array}{l}\text { Number of participants } \\
\text { without anxiety } \\
\text { symptom } \\
198\end{array}$} & \multirow{2}{*}{$\begin{array}{l}\text { Number of } \\
\text { participants with } \\
\text { anxiety symptom } \\
100\end{array}$} & \multirow{2}{*}{$\begin{array}{l}P \\
\text { value } \\
0.066\end{array}$} \\
\hline Marital status & Married/cohabitation & & & \\
\hline & $\begin{array}{l}\text { Unmarried/divorced } \\
\text { /widowed }\end{array}$ & 162 & 57 & \\
\hline \multirow[t]{5}{*}{ Working years } & $\leq 5$ years & 149 & 45 & \multirow[t]{5}{*}{0.089} \\
\hline & $6-10$ years & 61 & 34 & \\
\hline & $11-15$ years & 64 & 30 & \\
\hline & $15-20$ years & 32 & 17 & \\
\hline & 凶20 years & 54 & 31 & \\
\hline \multirow[t]{2}{*}{$\begin{array}{l}\text { Education } \\
\text { background }\end{array}$} & $\begin{array}{l}\text { Junior college or } \\
\text { below }\end{array}$ & 158 & 51 & \multirow[t]{2}{*}{0.015} \\
\hline & $\begin{array}{l}\text { Bachelor degree or } \\
\text { above }\end{array}$ & 202 & 106 & \\
\hline \multirow{2}{*}{$\begin{array}{l}\text { Professional } \\
\text { title }\end{array}$} & Junior & 247 & 102 & \multirow[t]{2}{*}{0.416} \\
\hline & $\begin{array}{l}\text { Medium-grade or } \\
\text { above }\end{array}$ & 113 & 55 & \\
\hline \multirow[t]{2}{*}{ leader } & yes & 16 & 7 & \multirow[t]{2}{*}{0.994} \\
\hline & no & 344 & 150 & \\
\hline \multirow{3}{*}{$\begin{array}{l}\text { Income } \\
\text { monthly(RMB) }\end{array}$} & $<5000$ & 56 & 12 & \multirow[t]{3}{*}{0.019} \\
\hline & $5000-10000$ & 284 & 140 & \\
\hline & $\$ 10000$ & 20 & 5 & \\
\hline \multirow{3}{*}{$\begin{array}{l}\text { Working hours } \\
\text { a week }\end{array}$} & $<40 \mathrm{H}$ & 199 & 61 & \multirow[t]{3}{*}{0.000} \\
\hline & $41-56 \mathrm{H}$ & 157 & 88 & \\
\hline & $\geq 56 \mathrm{H}$ & 4 & 7 & \\
\hline \multirow[t]{2}{*}{ Smoking } & Yes & 7 & 7 & \multirow[t]{2}{*}{0.107} \\
\hline & No & 352 & 150 & \\
\hline
\end{tabular}




\begin{tabular}{|c|c|c|c|c|}
\hline \multicolumn{2}{|c|}{ Demographic characteristics } & \multirow{2}{*}{$\begin{array}{l}\text { Number of participants } \\
\text { without anxiety } \\
\text { symptom } \\
24\end{array}$} & \multirow{2}{*}{$\begin{array}{l}\text { Number of } \\
\text { participants with } \\
\text { anxiety symptom } \\
8\end{array}$} & \multirow{2}{*}{$\begin{array}{l}P \\
\text { value } \\
0.514\end{array}$} \\
\hline $\begin{array}{l}\text { Alcohol } \\
\text { consumption }\end{array}$ & Yes & & & \\
\hline & No & 331 & 145 & \\
\hline \multirow[t]{3}{*}{ Regular diet } & Yes & 175 & 52 & \multirow[t]{3}{*}{0.003} \\
\hline & General & 144 & 76 & \\
\hline & No & 41 & 29 & \\
\hline \multirow[t]{3}{*}{$\begin{array}{l}\text { Physical } \\
\text { exercise }\end{array}$} & $\begin{array}{l}3 \text { or more times a } \\
\text { week }\end{array}$ & 28 & 7 & \multirow[t]{3}{*}{0.250} \\
\hline & 1-2 times a week & 100 & 39 & \\
\hline & No & 232 & 111 & \\
\hline \multirow{2}{*}{$\begin{array}{l}\text { Economic } \\
\text { stress }\end{array}$} & No & 126 & 36 & \multirow[t]{2}{*}{0.007} \\
\hline & Yes & 234 & 121 & \\
\hline \multirow{2}{*}{$\begin{array}{l}\text { Occupational } \\
\text { stress }\end{array}$} & No & 85 & 25 & \multirow[t]{2}{*}{0.050} \\
\hline & Yes & 275 & 132 & \\
\hline \multirow[t]{2}{*}{ Social stress } & No & 198 & 70 & 0.029 \\
\hline & Yes & 162 & 87 & \\
\hline
\end{tabular}


Table 3

Influencing factors analysis related to quality of life $(N=517)$

\begin{tabular}{|c|c|c|c|c|c|}
\hline Demographic characteristics & $\mathbf{N}$ & PCS & $P$ value & MCS & $P$ value \\
\hline \multicolumn{6}{|l|}{ Marital status } \\
\hline Married/cohabitation & 298 & $68.13 \pm 17.99$ & \multirow[t]{2}{*}{0.000} & $63.15 \pm 18.94$ & \multirow[t]{2}{*}{0.000} \\
\hline Unmarried/divorced/widowed & 219 & $76.41 \pm 16.48$ & & $70.33 \pm 16.97$ & \\
\hline \multicolumn{6}{|l|}{ Working years } \\
\hline$\leq 5$ years & 194 & $76.83 \pm 16.00$ & \multirow[t]{5}{*}{0.000} & $70.99 \pm 17.06$ & \multirow[t]{5}{*}{0.000} \\
\hline $6-10$ years & 95 & $65.66 \pm 16.83$ & & $59.94 \pm 17.80$ & \\
\hline $11-15$ years & 94 & $70.56 \pm 18.80$ & & $65.97 \pm 17.42$ & \\
\hline $15-20$ years & 49 & $69.49 \pm 17.33$ & & $61.23 \pm 21.45$ & \\
\hline$凶 20$ years & 85 & $68.89 \pm 19.19$ & & $65.30 \pm 18.94$ & \\
\hline \multicolumn{6}{|l|}{ Education background } \\
\hline Junior college or below & 209 & $75.11 \pm 16.83$ & \multirow[t]{2}{*}{0.000} & $70.71 \pm 16.83$ & \multirow[t]{2}{*}{0.000} \\
\hline Bachelor degree or above & 308 & $69.28 \pm 18.04$ & & $63.18 \pm 18.72$ & \\
\hline \multicolumn{6}{|l|}{ Professional title } \\
\hline Junior & 349 & $72.06 \pm 18.08$ & \multirow[t]{2}{*}{0.439} & $67.33 \pm 18.39$ & \multirow[t]{2}{*}{0.042} \\
\hline Medium-grade or above & 168 & $70.76 \pm 17.29$ & & $63.81 \pm 18.43$ & \\
\hline \multicolumn{6}{|l|}{ leader } \\
\hline Yes & 23 & $74.70 \pm 14.23$ & \multirow[t]{2}{*}{0.399} & $68.93 \pm 13.03$ & \multirow[t]{2}{*}{0.467} \\
\hline No & 494 & $71.49 \pm 17.97$ & & $66.06 \pm 18.67$ & \\
\hline \multicolumn{6}{|l|}{ Income monthly(RMB) } \\
\hline$<5000$ & 68 & $81.48 \pm 17.83$ & \multirow[t]{3}{*}{0.000} & $74.26 \pm 18.38$ & \multirow[t]{3}{*}{0.000} \\
\hline $5000-10000$ & 424 & $69.53 \pm 17.35$ & & $64.61 \pm 18.25$ & \\
\hline छ10000 & 25 & $80.59 \pm 14.02$ & & $70.96 \pm 16.14$ & \\
\hline \multicolumn{6}{|l|}{ Working hours a week } \\
\hline$<40 \mathrm{H}$ & 260 & $75.04 \pm 17.06$ & \multirow[t]{3}{*}{0.000} & $69.60 \pm 16.93$ & \multirow[t]{3}{*}{0.000} \\
\hline $41-56 \mathrm{H}$ & 245 & $68.89 \pm 17.81$ & & $63.43 \pm 18.88$ & \\
\hline$\geq 56 \mathrm{H}$ & 11 & $52.83 \pm 15.17$ & & $44.97 \pm 20.90$ & \\
\hline
\end{tabular}




\begin{tabular}{|c|c|c|c|c|c|}
\hline Demographic characteristics & $\mathbf{N}$ & PCS & $P$ value & MCS & $P$ value \\
\hline \multicolumn{6}{|l|}{ Smoking } \\
\hline Yes & 14 & $66.84 \pm 15.83$ & \multirow[t]{2}{*}{0.306} & $57.89 \pm 16.36$ & \multirow[t]{2}{*}{0.086} \\
\hline No & 502 & $71.80 \pm 17.88$ & & $66.47 \pm 18.46$ & \\
\hline \multicolumn{6}{|l|}{ Alcohol consumption } \\
\hline Yes & 32 & $72.75 \pm 14.17$ & \multirow[t]{2}{*}{0.764} & $60.90 \pm 17.38$ & \multirow[t]{2}{*}{0.103} \\
\hline No & 476 & $71.78 \pm 17.91$ & & $66.42 \pm 18.58$ & \\
\hline \multicolumn{6}{|l|}{ Regular diet } \\
\hline Yes & 227 & $74.92 \pm 17.73$ & \multirow[t]{3}{*}{0.000} & $69.19 \pm 18.58$ & \multirow[t]{3}{*}{0.000} \\
\hline General & 220 & $70.09 \pm 17.78$ & & $65.66 \pm 17.44$ & \\
\hline no & 70 & $65.84 \pm 16.28$ & & $58.14 \pm 18.90$ & \\
\hline \multicolumn{6}{|l|}{ Physical exercise } \\
\hline 3 or more times a week & 35 & $78.34 \pm 19.50$ & \multirow[t]{3}{*}{0.019} & $73.06 \pm 20.19$ & \multirow[t]{3}{*}{0.034} \\
\hline 1-2 times a week & 139 & $73.22 \pm 20.19$ & & $67.34 \pm 16.95$ & \\
\hline No & 343 & $70.31 \pm 17.70$ & & $65.02 \pm 18.74$ & \\
\hline \multicolumn{6}{|l|}{ Anxiety symptoms } \\
\hline No & 360 & $77.37 \pm 15.29$ & \multirow[t]{4}{*}{0.000} & $72.25 \pm 15.54$ & \multirow[t]{4}{*}{0.000} \\
\hline Mild & 116 & $62.12 \pm 15.21$ & & $55.59 \pm 15.58$ & \\
\hline Moderate & 34 & $48.79 \pm 15.31$ & & $44.31 \pm 18.14$ & \\
\hline severe & 7 & $45.52 \pm 11.02$ & & $36.51 \pm 16.71$ & \\
\hline \multicolumn{6}{|l|}{ Sleep disorder } \\
\hline Yes & 372 & $68.20 \pm 17.46$ & \multirow[t]{2}{*}{0.000} & $62.23 \pm 18.20$ & \multirow[t]{2}{*}{0.000} \\
\hline No & 145 & $80.44 \pm 15.64$ & & $76.33 \pm 14.98$ & \\
\hline \multicolumn{6}{|l|}{ Felling stress } \\
\hline Very heavy & 124 & $63.43 \pm 17.15$ & \multirow[t]{5}{*}{0.000} & $57.78 \pm 19.42$ & \multirow[t]{5}{*}{0.000} \\
\hline Heavy & 210 & $69.90 \pm 18.28$ & & $64.10 \pm 17.66$ & \\
\hline General & 175 & $78.91 \pm 14.56$ & & $73.84 \pm 15.38$ & \\
\hline Little & 4 & $78.53 \pm 11.99$ & & $84.24 \pm 9.27$ & \\
\hline Very little & 4 & $91.96 \pm 12.00$ & & $83.89 \pm 11.47$ & \\
\hline
\end{tabular}




\begin{tabular}{|c|c|c|c|c|c|}
\hline Demographic characteristics & $\mathbf{N}$ & PCS & $P$ value & MCS & $P$ value \\
\hline \multicolumn{6}{|l|}{ Economic stress } \\
\hline Yes & 355 & $70.04 \pm 17.60$ & \multirow[t]{2}{*}{0.002} & $64.41 \pm 18.61$ & \multirow[t]{2}{*}{0.001} \\
\hline No & 162 & $75.13 \pm 17.86$ & & $70.08 \pm 17.58$ & \\
\hline \multicolumn{6}{|l|}{ Occupational stress } \\
\hline Yes & 407 & $70.08 \pm 18.06$ & \multirow[t]{2}{*}{0.000} & $64.74 \pm 18.84$ & \multirow[t]{2}{*}{0.001} \\
\hline No & 110 & $77.40 \pm 15.68$ & & $71.56 \pm 15.95$ & \\
\hline \multicolumn{6}{|l|}{ Social stress } \\
\hline Yes & 249 & $69.12 \pm 17.29$ & \multirow[t]{2}{*}{0.002} & $63.24 \pm 18.79$ & \multirow[t]{2}{*}{0.000} \\
\hline No & 268 & $73.97 \pm 18.02$ & & $68.93 \pm 17.75$ & \\
\hline
\end{tabular}

Table 4

Correlation between sleep disorder and anxiety severity for nurses

\begin{tabular}{|lllll|}
\hline \multirow{2}{*}{ Anxiety severity } & \multicolumn{2}{l}{ NO. of subjects with Sleep disorder } & \multirow{2}{*}{$X^{2}$} & P value \\
\cline { 2 - 3 } & NO & YES & & \\
\hline No & 134 & 226 & 50.81 & 0.000 \\
\hline Mild & 11 & 105 & & \\
\hline Moderate & 0 & 34 & & \\
\hline severe & 0 & 7 & & \\
\hline
\end{tabular}


Table 5

logistic regression analysis of multiple factor influencing quality of sleep

\begin{tabular}{|lllllll|}
\hline Demographic characteristics & B & S.E. & P & OR & \multicolumn{2}{l|}{ 95\%Cl for OR } \\
\cline { 5 - 7 } & & & & & lower & upper \\
\hline Physical exercise & & & & & & \\
\hline 3 or more times a week & & & & 1.000 & & \\
\hline 1-2 times a week & -1.267 & 0.386 & 0.001 & 0.282 & 0.132 & 0.600 \\
\hline No & -0.008 & 0.239 & 0.972 & 0.992 & 0.620 & 1.585 \\
\hline Regular diet & & & & & & \\
\hline Yes & & & & 1.000 & & \\
\hline General & -1.484 & 0.417 & 0.000 & 0.227 & 0.100 & 0.513 \\
\hline no & -0.938 & 0.422 & 0.026 & 0.392 & 0.171 & 0.896 \\
\hline Income monthly(RMB) & & & & & & \\
\hline$<5000$ & & & & 1.000 & & \\
\hline $5000-10000$ & -1.021 & 0.504 & 0.043 & 0.360 & 0.134 & 0.966 \\
\hline घ10000 & 0.281 & 0.451 & 0.533 & 1.325 & 0.548 & 3.204 \\
\hline
\end{tabular}


Table 6

Logistic regression analysis of multiple factor influencing anxiety symptom

\begin{tabular}{|c|c|c|c|c|c|c|}
\hline \multirow[t]{2}{*}{ Demographic characteristics } & \multirow[t]{2}{*}{ B } & \multirow[t]{2}{*}{ S.E. } & \multirow[t]{2}{*}{$\mathbf{P}$} & \multirow[t]{2}{*}{ OR } & \multicolumn{2}{|c|}{$95 \% \mathrm{Cl}$ for OR } \\
\hline & & & & & lower & upper \\
\hline \multicolumn{7}{|l|}{ Education background } \\
\hline Junior college or below & & & & 1.000 & & \\
\hline Bachelor degree or above & 0.462 & 0.209 & 0.027 & 0.630 & 0.419 & 0.949 \\
\hline \multicolumn{7}{|l|}{ Working hours a week } \\
\hline$<40 \mathrm{H}$ & & & & 1.000 & & \\
\hline $41-56 \mathrm{H}$ & -1.550 & 0.651 & 0.017 & 0.212 & 0.059 & 0.760 \\
\hline$\geq 56 \mathrm{H}$ & -1.079 & 0.647 & 0.095 & 0.340 & 0.096 & 1.207 \\
\hline \multicolumn{7}{|l|}{ Regular diet } \\
\hline Yes & & & & 1.000 & & \\
\hline General & -0.719 & 0.304 & 0.018 & 0.487 & 0.269 & 0.884 \\
\hline no & -0.221 & 0.292 & 0.449 & 0.802 & 0.453 & 1.420 \\
\hline \multicolumn{7}{|l|}{ Economic stress } \\
\hline NO & & & & 1.000 & & \\
\hline YES & 0.503 & 0.227 & 0.027 & 0.605 & 0.388 & 0.944 \\
\hline
\end{tabular}

\section{Discussion}

\section{Prevalence and influencing factors of sleep disorder among nurses in tertiary hospitals}

Under the environment of long-term heavy workload, shift work system and high requirement of work quality, health care workers were lower status of physical and mental health than other groups. As a typical physical problem, Sleep disorder has become the focus of attention. According to our results and previous studies, the overall prevalence of sleep disorder in hospital staffs was higher than the general population [6, 37-40]. However, the status in nurses was even more serious. A pilot study in Urumqi(Xinjiang,China) using PSQI to evaluate the quality of sleep in physicians, indicated $59.92 \%$ participants had sleep disorders[41], that was lower than our results. The Other researcher in Tehran(Iran) and Taiwan(China) also revealed that nurses had higher incidence rate than other health care 
workers[42-43]. However, as a high-risk group, compare to other health care workers, nurses were most likely to suffer from sleep disorder that be focused first.

The previous study found highly stress correlated positively with sleep disorder[6, 44].In our study, nurses had sleep disorder with felling economic, occupational and social stress. Nurses in tertiary hospitals need deal with different kinds of medical condition, keep learning new medical knowledge and solving different social relationship problem. In fact, nurses always be exposed to high level stress for a long time[45-46].The percentage of nurses (working hours $₫ 40 \mathrm{H}$ per week) was $49.6 \%$ in our study. The previous studies indicated that heavy workload and longer working hours squeeze time for rest and exercise, and made nurses felling highly stress which influence quality of sleep[6]. However, along with the rapid rhythm of work and work time uncertainty, nurses develop a habit of irregular diet and exercise that affect the circadian rhythms which decrease the quality of life[2].

\section{Prevalence and influencing factors of anxiety symptoms among nurses in tertiary hospitals}

The prevalence of anxiety symptoms in nurses was $30.4 \%$ in our study that converged towards the results of similar studies[27, 47-49]. However, the prevalence in medical students and doctors were $12.5 \%$ and $21.1 \%$, respectively[50-51]. The previous studies indicated, nurses were risk group of anxiety symptom in health care workers. Comparing with other populations in China, the prevalence was obvious higher[5253]. According to the fact, it was warranted to in-depth studies and active interventions about anxiety symptoms in Chinese nurses.

Our study found higher education backgrounds was risk factor about anxiety symptom in nurses. The results of Liaoning general hospital also indicate nurses with higher education backgrounds may more likely be anxiety symptoms[27]. The results may reflect nurses with higher education backgrounds have higher learning and working target and demand. The previous study indicated nurses with regular meals have lower anxiety symptom(OR 0.719)that was similar with our study[27]. Keeping healthy lifestyle may be helpful for reducing anxiety symptoms in nurses. Longer working hours, heavier workload and lower salary make effort-reward imbalance in nurses that have negative effect for mental health and their job satisfaction[27, 54-55] however, a lot of researches have shown that the status of stress was significantly related to physical and mental health, including anxiety symptom[56-57].

\section{The correlation between sleep disorder and anxiety symptoms that influence the quality of life}

Several previous studies indicated sleep disorder have position relation with anxiety symptoms[58-59]. We also find the strong correlation between sleep disorder and anxiety symptom. We think there maybe correlation between physical health and mental health that the previous similar studies shown the same viewpoint[60].We also found nurses with sleep disorder or anxiety symptoms have lower quality of life, no matter PCS or MCS. The results may indicate that sleep disorder and anxiety symptom could respect poor quality of life. However, as the common influencing factors, stress, effort-reward imbalance and irregular lifestyle have significantly related to sleep disorder and anxiety symptom in our study. We should pay attention to these factors and talk steps to prevent these problems in nurses. 
The previous studies shown that nurses with higher social support have higher quality of life, higher quality of sleep and lower anxiety symptoms[27, 61-62]. However, providing social support like peer support, may be helpful for quality of sleep, relieve anxiety and increase quality of life in tertiary hospitals' nurses. The present results remanded hospital managers that need pay more attention on the effortreward balance by effective system like building appropriate performance measures. Meanwhile, by building positive channel like group activity, nurses could relieve the stress form job, economy and social. In addition, by healthy education, let nurses in tertiary maintain good lifestyle like keep regular diet and exercises. In other words, improving the quality of life, decreasing sleep disorder and anxiety symptoms must be on the agenda.

\section{Conclusion}

Hospital administrators had met challenges that nurses had bad sleep quality and anxiety symptoms because of heavy workload in tertiary hospitals in China. However, hierarchical diagnosis system engineering was carried out by National Health Commission of the People's Republic of China that relieve the pressure of tertiary hospitals' medical treatment, including doctors and nurses. In addition, from the perspective of hospital management, promotion of health lifestyle, effort-reward balance and providing social support may be significant to decrease the poor sleep quality and anxiety symptoms and increase quality of life in nurses.

\section{Limitation}

The limitation of this study was participants were concentrated in Beijing's tertiary hospitals. However, as the Chinese capital, high-quality medical resources are gathered in Beijing. By the rapid development of Chinese economy and traffic infrastructure construction, patients from all over China want to see a doctor in Beijing tertiary hospitals. It has imposed a heavy burden on medical resources in Beijing, including job burden in nurses. Ensuring physical and mental health of nurses in tertiary hospitals is more significant for Beijing medical system. However, we have plan to take a nationwide survey about quality of life in nurses.

\section{Abbreviations}

PSQI: Pittsburgh Sleep Quality Index; SAS: Zung Self-Rating Anxiety Scale; SF-36: 36-item Short Form Health Survey; PCS: physical component summary; MCS: mental component summary; OR:Odds ratio; ANOVA: analysis of variance

\section{Declarations}

\section{Ethics approval and consent to participate}


This study was approved by the Ethics Committee of Beijing Children hospital, Beijing Tongren Hospital, and Capital Medical University Affiliated Anzhen Hospital. All the participants were orally informed after our researchers had introduced the study. Written informed consent concerning conduct of the survey was obtained from each respondent before data collection. Confidentiality and privacy were maintained by using unique identification numbers instead of names.

\section{Consent for publication}

Not applicable

\section{Availability of data and material}

The datasets used and/or analyzed during the current study are available from the corresponding author on reasonable request.

\section{Competing interests}

The authors declare that they have no competing interests.

\section{Funding}

Financial support was provided by Key consulting projects of Chinese academy of engineering (No.2019XZ-29) and Hospital management foundation of Beijing Tongren hospital (NO.2016-YJJ-GLL-011). The funders had no role in the design of the study and collection, analysis, and in writing of the manuscript.

\section{Authors' contributions}

DW and $\mathrm{DH}$ designed the study and questionnaire. JL,MH and $\mathrm{HY}$ collected the data and control the quality of study. DW and YP analyze the date and wrote the manuscript. DH supervised the study and data analysis. All authors read and approved the manuscript.

\section{Acknowledgements}

We would like to acknowledge all the nurses for their kind cooperation from Beijing Children hospital, Beijing Tongren Hospital, and Capital Medical University Affiliated Anzhen Hospital. Special thanks to our researchers who took part in this study.

\section{Author details}

1. Beijing Tongren Hospital, NO.1 Dongjiaomin lane, DongCheng District, Beijing, China.

2. Capital Medical University Affiliated Anzhen Hospital, NO.2 Anzhen lane, ChaoYang District, Beijing, China

3. Department of public health, Capital Medical University, No.10, West gate, FengTai, District, Beijing, China 
*Corresponding author to De-min Han

\section{References}

1. Smart D,English A,James J,Wilson M,Daratha KB,Childers B,Magera C.Compassion fatigue and satisfaction: cross-sectional survey among US healthcare workers. Nurs Health Sci. $2014 ; 16(1): 3-10$.

2. Shoichi Asaoka, Sayaka Aritake, Yoko Komada, Akiko Ozaki, Yuko Odagiri, Shigeru Inoue,Teruichi Shimomitsu, and Yuichi Inoue. Factors Associated With Shift Work Disorder in Nurses Working With Rapid-Rotation Schedules in Japan: The Nurses' Sleep Health Project. Chronobiology International.2013;30(4): 628-636.

3. Eldevik MF, Flo E, Moen BE, Pallesen S, Bjorvatn B. Insomnia, Excessive Sleepiness, Excessive Fatigue, Anxiety, Depression and Shift Work Disorder in Nurses Having Less than 11 Hours inBetween Shifts. PLoS ONE.2013;8(8): e70882.

4. Hanne Irene Jensen, Jakob Markvart, René Holst, Tina Damgaard Thomsen, Jette West Larsen, Dorthe Maria Eg, Lisa Seest Nielsen. Shift work and quality of sleep: effect of working in designed dynamic light. Int Arch Occup Environ Health .2016; 89:49-61.

5. Chien PL, Su HF, Hsieh PC, Siao RY, Ling PY, Jou HJ. Sleep quality among female hospital staff nurses. Sleep Dis. 2013;2013:283490.

6. Hongyun Dong, Qiong Zhang, Zihua Sun, Fengxin Sang and Yingzhi Xu. Sleep disturbances among Chinese clinical nurses in general hospitals and its influencing factors. BMC Psychiatry.2017;17:241.

7. Fritschi L, Glass DC, Heyworth JS, Aronson K, Girschik J, Boyle T,Grundy A, Erren TC. Hypotheses for mechanisms linking shiftwork and cancer. Med Hypotheses.2011; 77:430-436.

8. Frost P, Kolstad HA, Bonde JP. Shift work and the risk of ischemic heart disease-a systematic review of the epidemiologic evidence. Scand J Work Environ Health.2009; 35:163-179.

9. Wang XS, Armstrong ME, Cairns BJ, Key TJ, Travis RC. Shift work and chronic disease: the epidemiological evidence. Occup Med.2011; 61:78-89.

10. Lisa F. Berkman, Sze Yan Liu, Leslie Hammer, Phyllis Moen, Laura Cousino Klein, Erin Kelly, Martha Fay, Kelly Davis, Mary Durham, Georgia Karuntzos, Orfeu M. Buxton. Work-family conflict, cardiometabolic risk and sleep duration in nursing employees. J Occup Health Psychol. 2015; 20(4): 420-433.

11. Mieda M, Sakurai T. Orexin (hypocretin) receptor agonists and antagonists for treatment of sleep disorders. Rationale for development and current status. CNS Drugs. 2013;27(2):83-90.

12. Rosado IV, Russo GH, Maia EM. Generating health elicits illness? The contradictions of work performed in emergency care units of public hospitals. Cien Saude Colet. 2015;20(10):3021-32.

13. D. M. Gaba and S. K. Howard. Fatigue among clinicians and the safety of patients, The New England Journal of Medicine.2002;347(16):1249-1255.

14. Weinger MB, Ancoli-Israel S. Sleep deprivation and clinical performance. JAMA.2002;287:955-957. 
15. Asken MJ, Raham DC. Resident performance and sleep deprivation: a review.J Med Educ. 1983;58:382-38.

16. Samkoff JS, Jacques $\mathrm{CH}$. A review of studies concerning effects of sleep deprivation and fatigue on residents' performance. Acad Med.1991;66:687-69.

17. Leung L, Becker CE. Sleep deprivation and house staff performance: update 1984-1991.J Occup Med.1992;34:1153-1160.

18. Owens JA. Sleep loss and fatigue in medical training. Curr Opin Pulm Med.2001;7:411-418.

19. National Institute of Mental Health. http://www.nimh.nih.gov/health/topics/anxietydisorder/index.shtml.

20. Eisenberg DM, Davis RB, Ettner SL,Appel S, Wilkey S, Van Rompay M. Trends in alternative medicine use in the United States,1990-1997:Results of a follow-up national survey.JAMA1998,280:15691575.

21. Phillips MR, Zhang J,Shi Q,Song Z,Ding Z,Pang S,Li X,Zhang Y, Wang Z. Prevalence, treatment, and associated disability of mental disorders in four provinces in China during 2001-05:an epidemiological survey. Lancet 2009,373:2041-2053.

22. Tuthill A, Slawik H, O'Rahilly S, Finer N. Psychiatric co-morbidities in patients attending specialist obesity services in the UK.QJM:Monthly Journal of the Association of Physicians 2006,99:317-325.

23. Ardekani ZZ, Kakooei H, Ayattollahi SM, Choobineh A, Aeraji GN.Prevalence of mental disorders among shift work hospital nurses in Shiraz,Iran.Pak J Biol Sci 2008,11:1605-1609.

24. Chan $\mathrm{AO}, \mathrm{Huak} \mathrm{CY}$.Influence of work environment on emotional health in a health care setting. Occup Med (Lond) 2004,54:207-212.

25. Mealer ML,Shelton A, Berg B, Rothbaum B, Moss M.Increased prevalence of post-traumatic stress disorder symptoms in critical care nurses. Am J Respir Crit Care Med 2007,175:693-697.

26. China health statistics yearbook in 2017.

27. Yu-Qin Gao, Bo-Chen Pan, Wei Sun, Hui Wu, Jia-Na Wang, Lie Wang.Anxiety symptoms among Chinese nurses and the associated factors: a cross sectional study. BMC Psychiatry. 2012;12:141

28. Jie Shen, Hairong Yu, YuanYuan Zhang, Anli Jiang. Professional quality of life: A cross-sectional survey among Chinese clinical nurses. Nursing and Health Sciences.2015;17,507-515.

29. Wong WS, Fielding R. The co-morbidity of chronic pain, insomnia, and fatigue in the general adult population of Hong Kong: prevalence and associated factors. J Psychosom Res. 2012;73(1):28-34.

30. Tsai PS, Wang SY, Wang MY, Su CT, Yang TT, Huang CJ, Fang SC. Psychometric evaluation of the Chinese version of the Pittsburgh sleep quality index(CPSQI) in primary insomnia and control subjects. Qual life Res. 2005; 14(8):1943-52.

31. Jegede RO. Psychometric attributes of the self-rating anxiety scale. Psychol Rep 1977, 40:303-306.

32. Olatunji BO, Deacon BJ, Abramowitz JS, Tolin DF. Dimensionality of somatic complaints: factor structure and psychometric properties of the self-rating anxiety scale. J Anxiety Disord 2006, 20:543-561. 
33. Michelson L, Mavissakalian M: Temporal stability of self-report measures in agoraphobia research. Behav Res Ther 1983, 21:695-698.

34. Liu XC, Oda S, Peng X, Asai K. Life events and anxiety in Chinese medical students. Soc Psychiatry Psychiatr Epidemiol. 1997, 32:63-67.

35. Faith Matcham, Sam Norton, Sophia Steer, Matthew Hotopf. Usefulness of the SF-36 Health Survey in screening for depressive and anxiety disorders in rheumatoid arthritis. BMC Musculoskeletal Disorders. 2016, 17:224.

36. Yang Zhang, Bo QU, Shi-si Lun, Ying Guo, Jie Liu3. The 36-Item Short Form Health Survey: Reliability and Validity in Chinese Medical Students. Int. J. Med. Sci. 2012, 9(7):521-526.

37. Wang L, Qin P, Zhao Y, Duan S, Zhang Q, Liu Y, Hu Y, Sun J. Prevalence and risk factors of poor sleep quality among Inner Mongolia medical university students: a cross-sectional survey. Psychiatry Res. 2016;244:243-8.

38. Li RH, Wing YK, Ho SC, Fong SY. Gender differences in insomnia-a study in the Hong Kong Chinese population. J Psychosom Res. 2002; 53(1):601-9.

39. Y. Doi, M. Minowa, M. Uchiyama, and M. Okawa. Subjective sleep quality and sleep problems in the general Japanese adult population. Psychiatry and Clinical Neurosciences. 2001,55(3):213-215.

40. W. S. Wong and R. Fielding. Prevalence of insomnia among Chinese adults in Hong Kong: a population-based study. Journal of Sleep Research.2011,20(1):117-126.

41. Xiaoyan Gao, Hua Ge, Yu Jiang, Yulong Lian, Chen Zhang, Jiwen Liu.Relationship between Job Stress and 5-HT2A Receptor Polymorphisms on Self-Reported Sleep Quality in Physicians in Urumqi (Xinjiang, China):A Cross-Sectional Study. Int. J. Environ. Res. Public Health. 2018, 15, 1034.

42. Ghalichi L, Pournik O, Ghaffari M, Vingard E. Sleep quality among health care workers. Arch Iran Med. 2013;16(2):100-3.

43. Tsai YC, Liu CH. Factors and symptoms associated with work stress and health-promoting lifestyles among hospital staff: a pilot study in Taiwan.BMC Health Serv Res. 2012;12:199.

44. Wilkins K, Beaudet MP. Work stress and health. Health Rep. 1998;10(3):47-62. (ENG); 49-66 (FRE).

45. Buddeberg-Fischer B, Klaghofer R, Stamm M, Siegrist J, Buddeberg C. Work stress and reduced health in young physicians: prospective evidence from Swiss residents. Int Arch Occup Environ Health. 2008;82(1):31-8.

46. Wheeler HH. A review of nurse occupational stress research: 1. Br J Nurs.1997;6(11):642-5.

47. Xu FM, Zhang JG, Lv LM: Investigation on psychological anxiety of clinical nursing staff. Chin Med Rec 2009, 10:25-27.

48. Xie XR: Study on relationship between personality, life events and social support and anxiety in nurses. Chin J of Pract Nurs 2009, 25:86-87.

49. Li SL, Li CH: Analysis on anxiety and its associated factors in nursing staff. Guide of China Med $2008,6: 141-142$. 
50. Liu XC, Oda S, Peng X, Asai K: Life events and anxiety in Chinese medical students. Soc Psychiatry Psychiatr Epidemiol 1997, 32:63-67.

51. Liu FY, Wang FX, Chen YX: A study of anxiety and its influencing factors in medical staff. Sichuan Mental Health 1998, 11:157-159.

52. Li ZJ, Zhou QH, Yu ZH: Life events, coping style and anxiety, depression of middle and elementary school teachers. Chin J of Clin Psychol 2003,11:285-286.

53. Yang GY, Li GH: Study on mental health status and education methods of college teachers. Mod Prev Med 2008, 35:1490-1492.

54. 54.Gao Y, Pan B: Comparative study on job satisfaction of Chinese and Japanese nurses. Chin Nurs Res 2008, 22:1147-1150.

55. Kikuchi Y, Nakaya M, Ikeda M, Narita K, Takeda M, Nishi M: Effort-reward imbalance and depressive state in nurses. Occup Med (Lond) 2010, 60:231-233.

56. Brigitte B. Jenull, Wolfgang Wiedermann. The different facets of work stress: A latent profile analysis of nurses' work demands. Journal of Applied Gerontology. 2015, 34(7):823-843.

57. Mark G, Smith AP: Occupational stress, job characteristics, coping, and the mental health of nurses. Br J Health Psychol 2012, 17:505-521.

58. Lee SO, Ahn SH, Kim MO: A study on women's fatigue and sleeping disturbance. Korean J Women Health Nurs 2005, 11(2):163-168.

59. Ayse Gulsen Teker, Nimet Emel Luleci. Sleep quality and anxiety level in employees. North Clin Istanb.2018,5(1):31-36.

60. Christine E Gould, Adam P Spira, Victoria Liou-Johnson, Erin Cassidy-Eagle, Makoto Kawai, Nehjla Mashal, Ruth O'Hara, Sherry A Beaudreau. Association of anxiety symptom clusters with sleep quality and daytime sleepiness. J Gerontol B Psychol Sci Soc Sci. 2018, 73(3): 413-420.

61. Tsai YC, Liu CH. Factors and symptoms associated with work stress and health-promoting lifestyles among hospital staff: a pilot study in Taiwan. BMC Health Serv Res. 2012;12:199.

62. Hope A, Kelleher CC, O'Connor M. Lifestyle practices and the health promoting environment of hospital nurses. J Adv Nurs. 1998;28(2):438-47. 